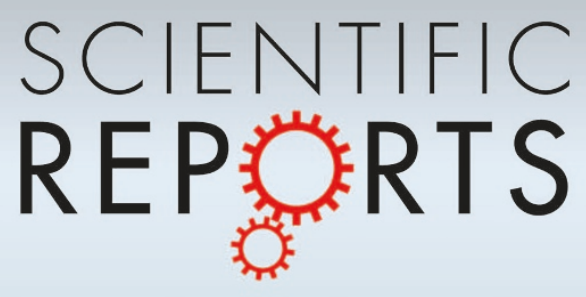

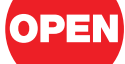

SUBJECT AREAS:

DNA METHYLATION

EPIGENETICS IN IMMUNE CELLS

B-CELL LYMPHOMA

PRE-CLINICAL STUDIES

Received

5 September 2012

Accepted

21 February 2013

Published

7 March 2013

Correspondence and requests for materials should be addressed to

A.M.W. (agata. wasik@ki.se)

\footnotetext{
* Current address:

Center for Primary
}

Health Care Research,

Lund University

Hospital, SE 20503

Malmö.

\section{SOXC transcription factors in mantle cell lymphoma: the role of promoter methylation in SOX 11 expression}

\author{
Agata Magdalena Wasik', Martin Lord' ', Xiao Wang1 *, Fang Zong' ', Patrik Andersson ${ }^{2}$, Eva Kimby ${ }^{3}$, \\ Birger Christensson' ${ }^{1}$, Mohsen Karimi ${ }^{3} \&$ Birgitta Sander $^{1}$
}

'Department of Laboratory Medicine, Division of Pathology, Karolinska Institutet and Karolinska University Hospital, SE 14186 Stockholm, ${ }^{2}$ Department of Hematology, Stockholm South Hospital, SE 11883 Stockholm, ${ }^{3}$ Division of Hematology, Department of Medicine, Karolinska Institutet, Karolinska University Hospital, SE 14186 Stockholm.

The related transcription factors SOX11, SOX4 and SOX12 (classified as the SOXC family) compete for the same target genes. SOX11 is expressed in most mantle cell lymphomas (MCL) but a small subset is, like normal lymphocytes, SOX11 negative. Here we report the variable expression of SOX4 and high expression of SOX12 in MCL compared to non-malignant tissue. Our results show that the expression of the SOXC genes is highly correlated in SOX11 positive MCL. SOX11 expression is epigenetically regulated but there are partly conflicting results regarding the underlying mechanisms. Here we report that the SOX11 promoter region is hypomethylated in both MCL and normal B-lymphocytes. Methylation at other sites is important for sustaining high SOX11 in MCL since treatment with 5-azacytidine decreased SOX11 levels in SOX11 positive MCL cell lines: Granta519 and Rec1. Furthermore, 5-azacytidine treatment of the SOX11 negative MCL cell line, JVM2, induced SOX4 but not SOX11.

s he neuronal transcription factor Sry-related high-mobility-group box 11 (SOX11) is normally expressed during embryogenesis and is critical for survival of neuronal and mesenchymal progenitor cells ${ }^{1}$. SOX11, SOX4 and SOX12 constitute the SOXC family of transcription factors that compete for certain transcriptional targets in vitro ${ }^{2}$ and have partly overlapping functions in vivo ${ }^{1}$. SOX4 is of importance for B cell development ${ }^{3}$ while the role of SOX 11 and SOX12 in hematopoiesis remains largely unknown. SOX11 was recently found to be expressed in mantle cell lymphoma (MCL) and in subsets of hairy cell leukemias, Burkitt lymphomas, B cell lymphoblastic leukemias but not in other B cell lymphomas or in normal B lymphocytes ${ }^{4-7}$. Importantly, a small subset of MCL lacks expression of SOX11 and these have been suggested to differ from SOX11 positive MCL in several aspects: SOX11 negative MCL harbor less chromosomal aberrations and hypermutated immunoglobulin receptor genes ${ }^{8}$ but are also more often $\mathrm{p} 53$ positive by immunohistochemistry ${ }^{9}$. SOX 11 has also been suggested to be of prognostic importance in $\mathrm{MCL}^{7,8,10}$ but recent studies have indicated that SOX11 cannot be used as a predictive marker for aggressive or indolent disease 9 .

MCL harbour high number of genetic aberrations but none involving the SOX11 genomic region at chromosome $2 \mathrm{p} 25^{11}$. Instead, epigenetic mechanisms have been suggested to regulate SOX11 expression in MCL. Gustavsson et al. ${ }^{12}$ suggested that promoter methylation is responsible for the differential expression of SOX11 in various types of lymphomas. Interestingly, low methylation of the SOX11 promoter was found both in SOX11 positive cell lines $(n=6)$ and primary MCL $(n=4)$, as well as in SOX11 negative MCL cell lines $(n=2)$. High level of SOX11 promoter methylation was described in follicular lymphoma (3 cell lines and 5 primary FL) and diffuse large B cell lymphoma (4 cell lines and 1 primary DLBCL) ${ }^{12}$. In contrast, a thorough investigation of SOX11 expression during lymphoid development and in various lymphoid malignancies suggested that SOX11 is regulated by histone modifications rather than promoter methylation ${ }^{13}$.

In this study we investigate if the levels of promoter methylation correlate to SOX11 expression in MCL. The other members of the SOXC group of transcription factors, SOX4 and SOX12, were also investigated in MCL and non-malignant lymphocytes.

\section{Results}

Expression of SOX11, SOX4 and SOX12 in MCL and non-malignant lymphoid tissue. We investigated SOX11 expression by immunohistochemistry in primary cyclin D1 positive MCL, all carrying the $\mathrm{t}(11 ; 14)(\mathrm{q} 13 ; \mathrm{q} 32)$, and in non-malignant tissue from tonsil, lymph node and spleen. The characteristics of the primary MCL cases and 
the non-malignant tissue are presented in Table 1 . Nuclear positivity for SOX11 was detected in 24/27 primary MCL cases that could be immunohistochemically analyzed while 3 cases (MCL-21, MCL-22 and MCL-23) and the non-malignant lymphoid tissue lacked SOX11 expression (Table 1 and supplementary Figure S1).

Based on the current literature, suggesting partially overlapping functions of the SOXC transcription factors, we hypothesized that SOX11 negative MCL may highly express other SOXC genes. It was therefore of interest to investigate the mRNA levels of the SOXC transcription factor family members SOX11, SOX4 and SOX12, in the primary MCL cases, in the MCL cell lines Granta519, Rec1, JeKo and JVM2 and in the non-malignant lymphoid tissues from lymph node, spleen and tonsil (Figure la, b, c). The mRNA expression levels in MCL and non-malignant tissue were normalized to tonsil B cells. A high expression of SOX11 (median 500-fold compared to nonmalignant B cells) was detected in most of the primary MCL. The three primary MCL (MCL-21, MCL-22 and MCL-23) that lacked SOX11 protein expression by immunohistochemistry had very low levels of SOX11 mRNA (SOX11 negative MCL). Two of these patients had a leukemic disease with bone marrow involvement and a clinically indolent disease presentation, while the third case was an aggressive blastoid variant of MCL. Analysis of SOX4 mRNA levels showed that 13/29 MCL cases had lower expression levels than the non-malignant tissues, 6/29 had comparable SOX4 mRNA levels to non-malignant tissues, while 10/29 MCL cases had higher SOX4 mRNA levels than tonsil B cells. SOX12 mRNA in 2/29 samples was comparable to tonsil B cells while in the remaining $27 \mathrm{MCL}$ samples SOX12 mRNA was expressed 2- to 22- fold more than in nonmalignant B cells. The three SOX11 negative MCL cases, MCL-21, MCL-22 and MCL-23, expressed SOX12. The expression of SOXC genes in MCL cell lines is varied. Granta519, Recl and JeKo carried
860, 60 and 550 times higher SOX11 mRNA than non-malignant B cells, while SOX11 was not detected in the JVM2 cell line. The levels of SOX4 mRNA in Granta519 and Rec1 was comparable to nonmalignant B cells, while in JeKo SOX4 was nearly 15 times upregulated. The SOX4 level was 10 times lower in JVM2 than in control B cells. In Granta519, Rec1 and JVM2 the SOX12 levels were approximately 3 times higher than in non-malignant B cells, while JeKo overexpressed SOX12 around 35 times. Thus, among cell lines tested only JeKo had increased mRNA levels of all SOXC genes. We also looked at the SOXC mRNA levels in MCL in relation to the housekeeping gene $\beta$-actin (presented as delta $\mathrm{Ct}$ ) to compare the mRNA of SOXC genes between each other in every sample. The mRNA levels within each primary sample showed that 20/26 SOX11-positive MCL express higher or comparable absolute levels of SOX11 mRNA (thus as lower delta Ct value) than SOX4 and SOX12. All SOX11-negative MCL and non-malignant B cells expressed higher absolute mRNA levels of SOX4 and SOX12 than SOX11 (supplementary Figure S2).

We also investigated whether there was any correlation between the expression levels of the three SOXC genes. When this was tested in all MCL and non-malignant cases, SOX4 and SOX11 and SOX12 and SOX11 expression levels were moderately correlated (correlation coeff. 0,$48 ; \mathrm{p}<0,05$ and 0,$50 ; \mathrm{p}<0,05$, respectively) and there was no significant correlation between SOX12 and SOX4 (correlation coeff. $0,24, \mathrm{p}<0,5$ ) (supplementary Figure S2). However, among SOX11 positive cases we found a highly significant correlation between SOX4 and SOX11 (Spearman's correlation coefficient: 0,56; $\mathrm{p}<0,005$ ), SOX12 and SOX11 (Spearman's correlation coefficient: 0,$63 ; p<0,005)$ and moderate correlation between SOX12 and SOX4 mRNA (Spearman's correlation coefficient: 0,40; p < $0,005)$.

Table 1 | Characteristics of primary samples from patients with $\mathrm{MCL}$

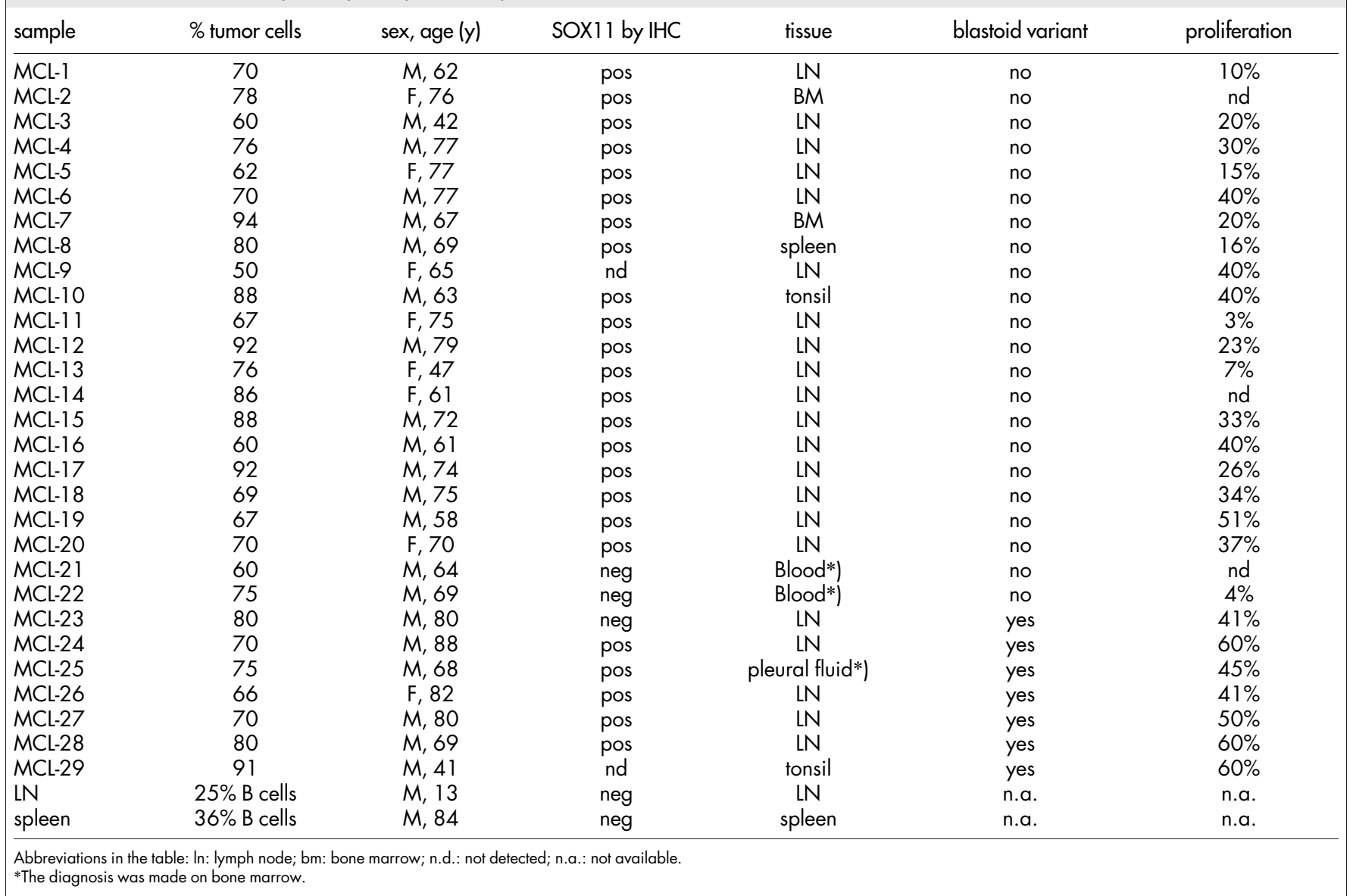




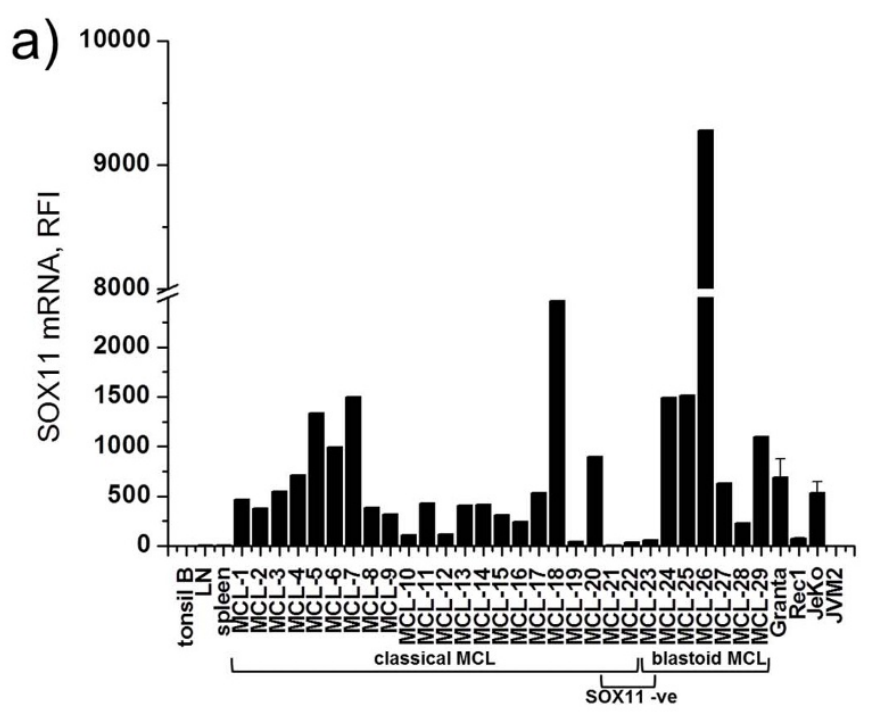

b)
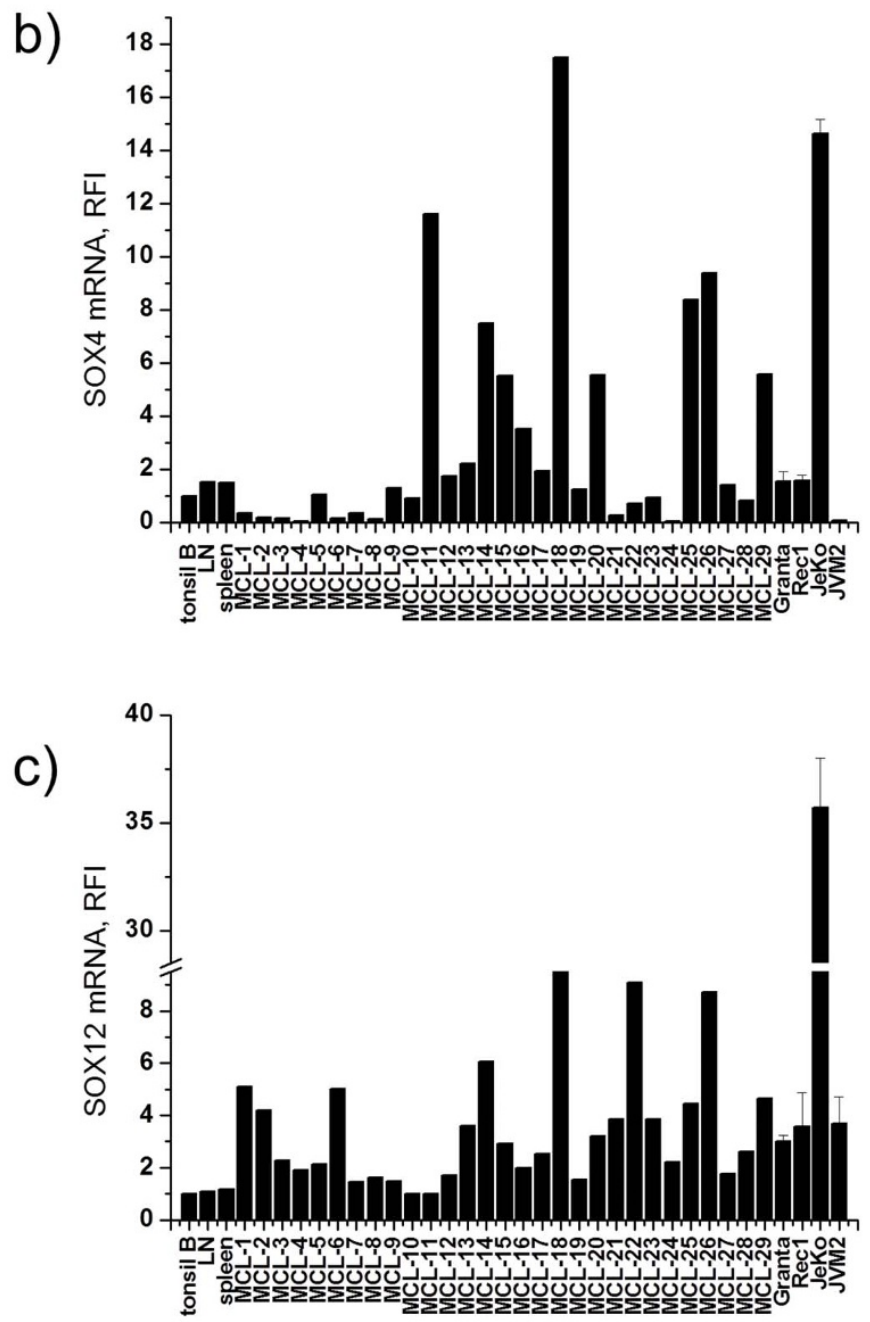

Figure 1 Expression of SOXC mRNA in MCL. Expression levels of (a) SOX11, (b) SOX4 , (c) SOX12 mRNA levels in MCL primary samples, MCL cell lines and non-malignant tissue. Expression levels were normalized to tonsil B cells.

The level of SOX11 expression in MCL is not dependent on promoter methylation. In order to investigate how promoter methylation correlate to SOX11 expression in MCL, primers targeting four different $\mathrm{CpG}$ rich fragments of the SOX11 5 'flanking region were used (Figure $2 \mathrm{a}$ below the chart and
Supplementary Figure S3). Fragment $1(-954$ to -637$)$ contained 69 unique CpG sites, fragment $2(-343$ to -33$)$ contained $50 \mathrm{CpG}$ sites, both fragments are located in the promoter region. Fragment 3 ( -52 to 323 ), located in mRNA start region, contained $52 \mathrm{CpG}$ sites, three of which were overlapping with fragment 2. Fragment 4 (384 to 784 ), in the mRNA start region, contained 40 unique CpG sites. Fragment 2 was partly overlapping (14 CpG sites) with the region previously described to be determinative for SOX11 expression in lymphoma cell lines ${ }^{12}$ (for primer design see supplementary Figure S3). The DNA was treated with the McrBC endonuclease, digesting DNA at methylation sites. McrBC-digested DNA results in higher Ct values. The difference in $\mathrm{Ct}$ values $(\Delta \mathrm{Ct})$ between McrBC-treated and mock-treated DNA thus corresponds to the number of methylated CpGs within the amplified fragment. Fragment 1 contained partly methylated CpGs. The methylation of the other fragments was varied (Figure 2a). Within the entity of MCL there was no correlation between SOX11 expression pattern and the methylation status of the amplified fragments 1,2, 3 and 4 of the 5 'flanking region of SOX11. We also used the bisulfite sequencing approach to verify the promoter methylation status within the MCL cases investigated. For this we used the primers designed by Vegliante et al. and analyzed the first four CpG sites since these were the most informative ${ }^{13}$. The vast majority of MCL cases and MCL cell lines, as well as non-malignant B-lymphocytes and lymphoid tissue, showed low methylation of the investigated region of the SOX11 promoter (Figure 2b). Also the SOX11 negative primary MCL cases MCL-21 and MCL-23 and JVM2 were hypomethylated. The SOX11 negative MCL-22 and the SOX11 negative Burkitt lymphoma cell line Raji (the latter used as a control) were hypermethylated compared to the other MCL cases and non-malignant lymphoid tissue (Figure $2 b$ ).

5-azacitidine treatment in MCL cell lines causes decrease of SOX11 expression via SOX11 promoter methylation-independent mechanisms. MCL cell lines were treated with $1 \mu \mathrm{M}$ of the DNA methyltransferase inhibitor 5-azacitidine (5-AZA) for 6 days. Granta519, Rec1, JeKo and JVM2 cells were re-cultured in fresh medium containing fresh 5-AZA every 48 hours. Cell cycle analysis after 6 days of 5-AZA treatment showed that cells accumulated in the sub-G1 upon 5-AZA treatment (data not shown). No increase in SOX11 mRNA expression was detected in the SOX11 negative MCL cell line JVM2 or in the SOX11 positive cell lines throughout the treatment with 5-AZA (Figure 3a). Instead, in Granta519 and Rec1, mRNA and protein levels decreased upon 5-AZA treatment (Figure 3d).

We investigated whether treatment with 5-AZA influenced the mRNA expression of SOX4 and SOX12. In contrast to SOX11, SOX4 mRNA remained unchanged in Granta519, Rec1 and JeKo cell lines after 5-AZA treatment. However, SOX4 levels in JVM2 cell line increased 23-fold after 6 days of incubation with 5-AZA (Figure 3b). SOX12 mRNA levels remained unchanged (Figure 3c).

\section{Discussion}

We investigated SOX11 protein and SOX11, SOX4 and SOX12 mRNA expression levels in 29 cases of MCL and in non-malignant tissue from tonsil, lymph node and spleen. Among MCL primary cases 26/29 were SOX11 positive and 3/29 (MCL-21, MCL-22 and MCL-23) and the non-malignant lymphoid tissue were SOX11 negative. Interestingly, the expression of the three SOXC genes was positively correlated in SOX11 positive MCL, suggesting that the expression of the SOXC genes may be co-regulated.

We studied the potential dependence between the SOX11 expression and SOX11 promoter methylation. Our results confirm the findings by Vegliante et al. in that we find the SOX11 promoter region hypomethylated in both SOX11 positive primary MCL cases and the SOX11 negative case MCL-21 and MCL-23 as well as in all 
a)
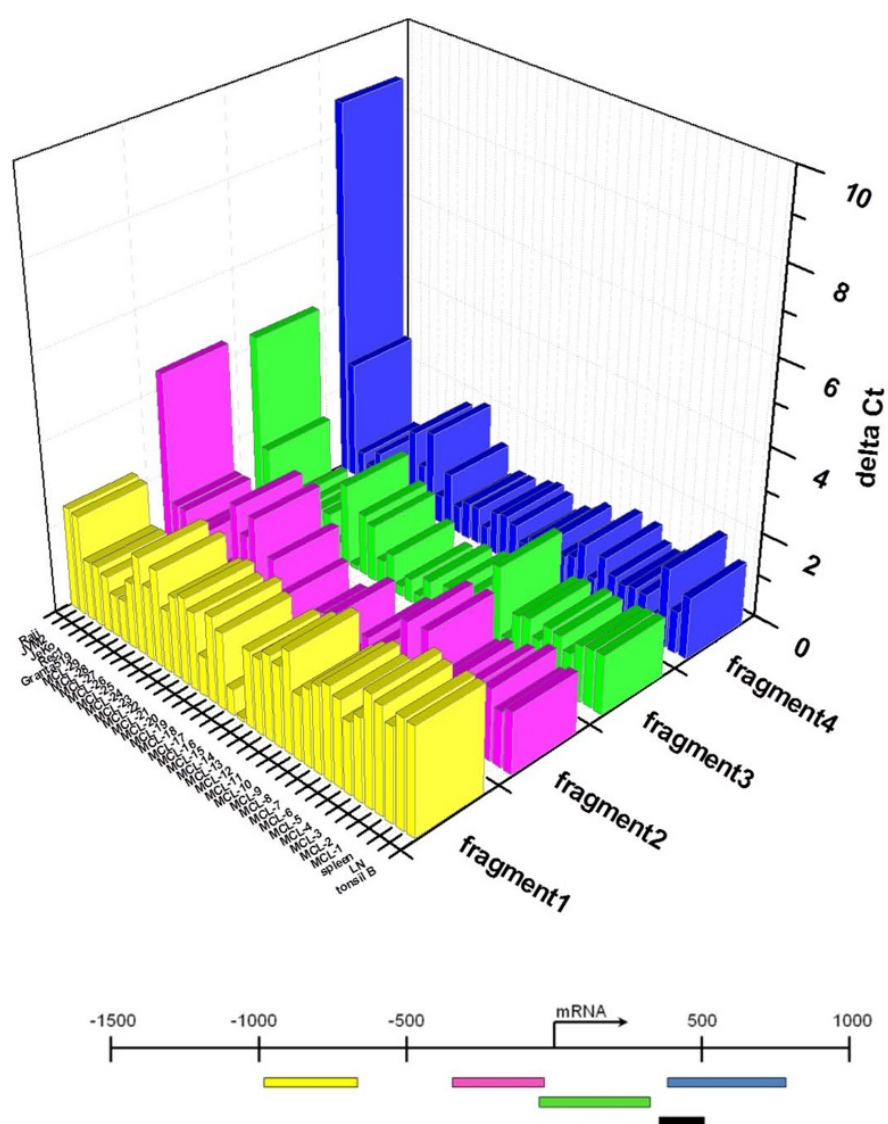

b)

\begin{tabular}{|c|c|c|c|c|}
\hline & CpG1 & CpG2 & CpG3 & CpG4 \\
\hline tonsil B & 7 & 7 & 18 & 13 \\
\hline LN & 5 & 5 & 16 & 10 \\
\hline spleen & 15 & 15 & 28 & 22 \\
\hline MCL-1 & 4 & 4 & 7 & 7 \\
\hline MCL-2 & 2 & 2 & 4 & 6 \\
\hline MCL-3 & 4 & 4 & 8 & 8 \\
\hline MCL-4 & 5 & 5 & 10 & 8 \\
\hline MCL-5 & 5 & 5 & 10 & 9 \\
\hline MCL-6 & 3 & 3 & 5 & 6 \\
\hline MCL-7 & 2 & 2 & 3 & 5 \\
\hline MCL-8 & 4 & 4 & 4 & 8 \\
\hline MCL-9 & 7 & 6 & 13 & 8 \\
\hline MCL-10 & 7 & 7 & 9 & 8 \\
\hline MCL-11 & 16 & 17 & 24 & 19 \\
\hline MCL-12 & 4 & 3 & 5 & 4 \\
\hline MCL-13 & 3 & 3 & 7 & 4 \\
\hline MCL-14 & 3 & 2 & 3 & 3 \\
\hline MCL-15 & 6 & 6 & 10 & 7 \\
\hline MCL-16 & 3 & 3 & 6 & 4 \\
\hline MCL-17 & 4 & 3 & 6 & 4 \\
\hline MCL-18 & 3 & 3 & 5 & 4 \\
\hline MCL-19 & 6 & 5 & 9 & 7 \\
\hline MCL-20 & 5 & 5 & 9 & 6 \\
\hline MCL-21 & 3 & 3 & 7 & 8 \\
\hline MCL-22 & 38 & 27 & 54 & 53 \\
\hline MCL-23 & 6 & 5 & 8 & 7 \\
\hline MCL-24 & 2 & 2 & 3 & 5 \\
\hline MCL-25 & 2 & 2 & 2 & 5 \\
\hline MCL-26 & 3 & 4 & 6 & 7 \\
\hline MCL-27 & 3 & 2 & 3 & 3 \\
\hline MCL-28 & 4 & 3 & 5 & 5 \\
\hline MCL-29 & 3 & 2 & 2 & 3 \\
\hline Granta519 & 2 & 2 & 3 & 4 \\
\hline Rec1 & 5 & 5 & 9 & 10 \\
\hline JeKo & 1 & 2 & 2 & 4 \\
\hline JVM2 & 5 & 4 & 7 & 7 \\
\hline Raji & 56 & 67 & 92 & 95 \\
\hline & & & & \\
\hline
\end{tabular}

Figure $2 \mid$ The SOX11 promoter methylation level in MCL. (a) methylation level of SOX11 promoter in MCL cases, cell lines and non-malignant cells shown by the $\Delta \mathrm{Ct}$ values corresponding to the difference between McrBC-digested and non-digested DNA sequence of four fragments within the SOX11 promoter; the relative location of different fragments is presented beneath the chart on the color-coded graph, black block presents location of pyroprimers. (b) SOX11 promoter methylation shown by pyrosequencing. The Raji (Burkitt lymphoma) cell line with a hypermethylated SOX11 promoter was used as a control for high methylation.

MCL cell lines tested. Only the SOX11 negative MCL case MCL-22 showed some degree of methylation. Importantly, the SOX11 promoter region was also hypomethylated in non-malignant B-lymphocytes and reactive lymphoid tissue, lacking SOX11 expression.

In spite of the fact that only low levels of methylation of SOX11 was found it was considered of interest to analyze the effect of demethylating agents on the expression of SOXC genes. The decrease in SOX11 mRNA and protein levels in 5-AZA treated Granta519 and Rec1 MCL cell lines and the fact that the SOX11 promoter region is already hypomethylated in MCL (current data and ${ }^{12,13}$ ) suggest that effect of 5-AZA on SOX11 levels might be indirect. Promoter methylation-independent effects of 5-AZA on certain genes were already reported in $\mathrm{MCL}^{15}, \mathrm{AML}^{16}$, colon cancer ${ }^{17}$ and in breast cancer ${ }^{18}$. Vegliante et al. showed that the histone deacetylase (HDAC) inhibitor SAHA induced SOX11 expression in JVM2 and Raji cell lines. Treatment of Raji cells with SAHA at $5 \mu \mathrm{M}$ markedly increased SOX11 mRNA levels, while the co-treatment with SAHA and $1 \mu \mathrm{M} 5$-AZA was less efficient, suggesting that 5-AZA might negatively influence SOX11 expression ${ }^{13}$ in line with our results. Moreover, SOXC group gene regulation may include microRNAs. In endomet- 

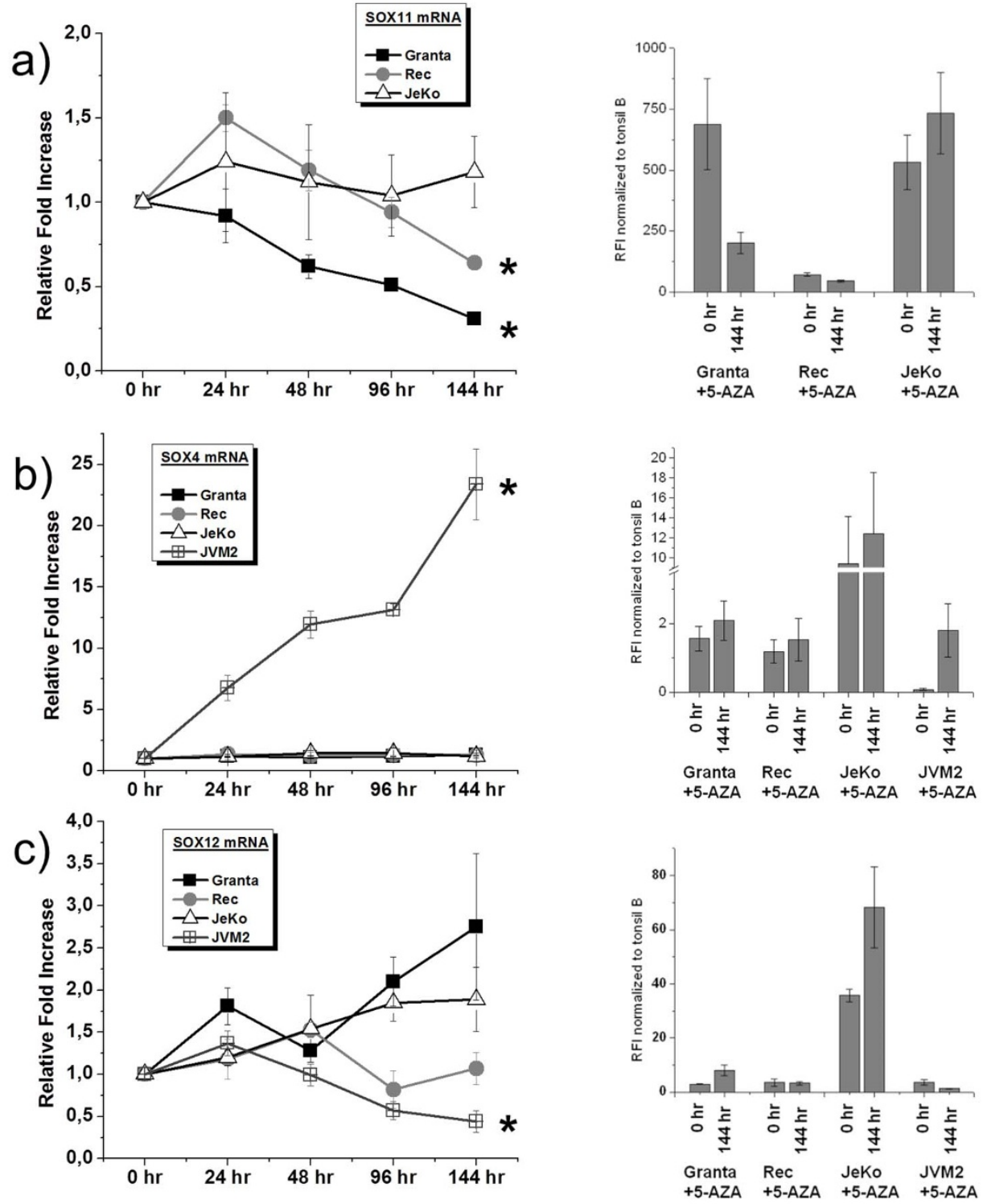

d)

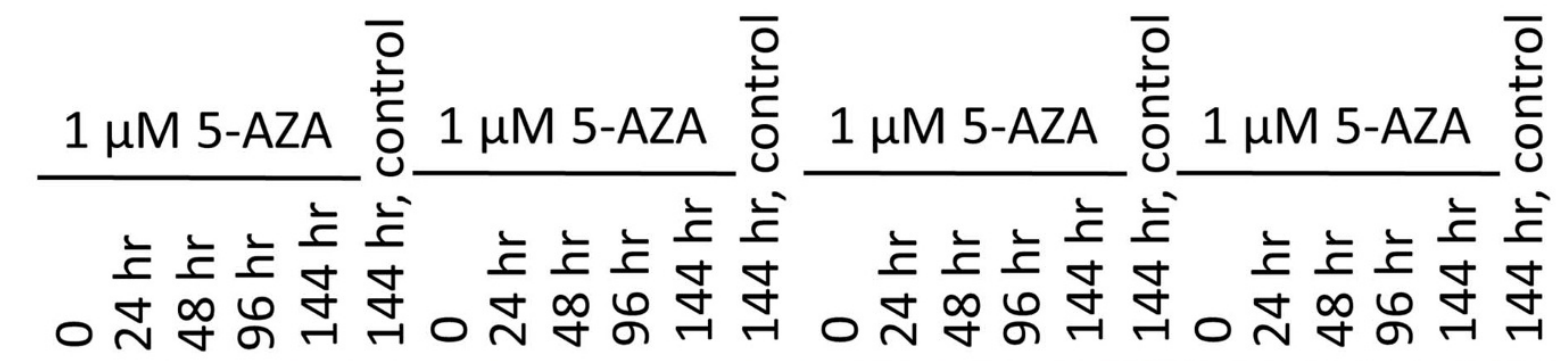

\section{sox11}

$\beta$-actin
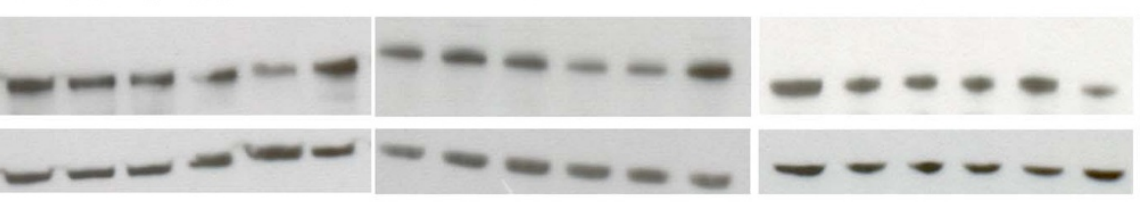

\section{Granta519}

Rec1

JeKo

JVM2

Figure 3 Expression of the SOXC group of transcription factors in 5-azacitidine-treated MCL cell lines. mRNA expression levels ( \pm SEM) of (a) SOX11, (b) SOX4, (c) SOX12 in MCL cell lines treated with $1 \mu \mathrm{M} 5$-azacitidine for 6 days expressed as relative fold change normalized to gene levels at 0 time point (left panel) or normalized to SOX11/SOX4/SOX12 gene expression in non-malignant tonsil B (right panel). * indicates significant changes in SOXC expression by Student's $t$ test $(\mathrm{p}<0,05, \mathrm{n}=3) \mathrm{d}$ ) SOX11 protein expression in MCL cell lines treated with 5-azacytidine for different time periods. Exposure times were different for the different cell lines and optimized in order to visualize differences in SOX11 expression over time. 
rial and gastric cancer SOX4 is regulated by miR-129-2 $2^{19,20}$. The potential role of microRNA in the regulation of SOX11 expression in MCL is however largely unknown.

5-AZA treatment has global effects on methylation and gene expression and may not be the ideal means to study the expression of single genes. We do however find it interesting that the SOX11negative JVM2 cell line expressed higher levels of SOX4 and lower levels of SOX12 mRNA levels after 5-AZA treatment.

In conclusion our results demonstrate that the SOX11 promoter region is hypomethylated both in SOX11 positive MCL and in SOX11 negative non-malignant lymphoid tissue and heterogeneously methylated in SOX11 negative primary MCL cases. The expression of SOX11 in MCL is therefore regulated by other mechanisms than promoter methylation as recently suggested by Vegliante et al. ${ }^{13}$. Furthermore, we demonstrate that another member of the SOXC group of transcription factors, SOX12, is overexpressed in both SOX11 positive and SOX11 negative MCL compared to nonmalignant lymphocytes. The third member of the SOXC family, SOX4, is generally less expressed in most MCL compared to nonmalignant B-lymphocytes but could be induced by 5-AZA in the SOX11 negative MCL cell line JVM2. In primary SOX11 positive MCL the expression of the SOXC group of genes were positively correlated, suggesting common regulatory mechanisms. The role of the SOXC group of genes in lymphoma pathogenesis is largely unknown. Since this group of transcription factors have been shown to have partly overlapping functions in gene regulation in vitro and during embryogenesis the differential expression in MCL might have important, but yet not well characterized, functions in MCL biology.

\section{Methods}

Reagents. 5-azacitidine (Sigma-Aldrich) was diluted in cell culture medium to $500 \mu \mathrm{M}$ concentration, aliquoted and kept at $-80^{\circ} \mathrm{C}$ until used.

Patient samples and control tissues and cells. Cells were isolated as described previously ${ }^{14}$, viability frozen in DMSO according to the biobank procedures at Karolinska University Hospital Huddinge and stored in $-135^{\circ} \mathrm{C}$ or the cell pellet was frozen at $-80^{\circ} \mathrm{C}$. Non-malignant $\mathrm{B}$ cells from a hyperplastic tonsil were purified by magnetic bead separation. After separation the sample contained $80 \%$ of B cells.

Cell culture. Granta519, Rec1, JeKo, JVM2 MCL cell lines and primary cells were obtained and cultured as previously described ${ }^{14}$. Prior to setting up experiments, cells were cultured overnight (cell lines) in fresh RPMI + GlutaMax (Invitrogen) with 10\% FBS (Invitrogen). The cell concentration at the start of each experiment was $0.5 \times 10^{6} / \mathrm{ml}$. Cells were counted and recultured with $1 \mu \mathrm{M} 5$-AZA every 48 hours.

RNA isolation and cDNA synthesis. RNA was isolated using the RNeasy Plus mini kit (Qiagen) according to the manufacturer's protocol. Quantification and quality of the RNA preparations were measured using the NanoDrop ND-1000 spectrophotometer (Saveen Werner). Complementary DNA (cDNA) was synthesized using the Omniscript Reverse Transcription (RT) kit (Qiagen) according to manufacturer's protocol. RNaseOut Recombinant Ribonuclease inhibitor and the Oligo dT primers used were purchased from Invitrogen.

Quantitative PCR (qPCR). mRNA expression levels of SOX4, SOX11 and SOX12 were assessed by Real-Time PCR using Platinum SYBR Green qPCR Supermix-UDG (Invitrogen) according to the manufacturer's protocol. Primer sequences (Eurogentec) for the selected genes are shown in Supplementary Table 1. Each sample was prepared in triplicates in a 96 well plate (BioRad) and the reactions were performed with the $\mathrm{C} 1000 \mathrm{Thermal}$ cycler (BioRad). An initial step was performed at $95^{\circ} \mathrm{C}$ for $2 \mathrm{~min}$, followed by 40 cycles of $95^{\circ} \mathrm{C}$ for 15 seconds and finished by $57^{\circ} \mathrm{C}$ for $30 \mathrm{sec}$. The results were analyzed and cycle threshold $(\mathrm{Ct})$ values of transcripts were quantified using CFX manager software (BioRad). The $\Delta \mathrm{Ct}$ values were calculated using $\beta$-actin as reference. Tonsil $\mathrm{B}$ cells were used as control to calculate the $\Delta \Delta \mathrm{Ct}$ value and to determine the relative fold increase (RFI).

DNA extraction and McrBC-based methylation assay. DNA was isolated using Gen-Elute Mammalian Genomic DNA MiniPrep Kit (Sigma Aldrich) according to the manufacturer's protocol. 800 ng DNA was incubated with the mastermix containing McrBC or mock-mastermix (according to the manufacturer's guidance, New England Biolabs) for 2 hours at $37^{\circ} \mathrm{C}$, followed by $15 \mathrm{~min}$ at $65^{\circ} \mathrm{C}$. Then the selected DNA fragments (Supplementary Table S1) were amplified by qPCR using SYBR Green PCR Master Mix (Applied Biosystems). Each sample was prepared in duplicates in a 96 well plate (BioRad) and the reactions were performed with the $\mathrm{C} 1000$ Thermal cycler (BioRad). An initial step was performed at $95^{\circ} \mathrm{C}$ for $2 \mathrm{~min}$, followed by 40 cycles of $95^{\circ} \mathrm{C}$ for $15 \mathrm{sec}, 56^{\circ} \mathrm{C}$ for $30 \mathrm{sec}$ and $72^{\circ} \mathrm{C}$ for $30 \mathrm{sec}$. The results were analyzed and cycle threshold $(\mathrm{Ct})$ values of transcripts were quantified using CFX manager software (BioRad). The $\Delta \mathrm{Ct}$ values were calculated as a difference between Ct of McrBC-treated versus mock-treated DNA.

Bisulfite treatment and pyrosequencing analysis. The bisulfite treatment was performed using the kit EZ DNA Methylation-Gold Kit (Zymo Research) according to the manufacturer's protocol. Pyrosequencing was performed using PyroMark Q24 system (Qiagen). Proteinase K was from Sigma Aldrich.

Western blotting. Cells were harvested, washed in PBS and lysed in ice-cold RIPA buffer (Sigma Aldrich) supplemented with protease inhibitors cocktail III (Sigma Aldrich). Protein concentrations were determined using bicinchoninic acid (BCA) assay according to manufacturer's protocol (Pierce). $30 \mu \mathrm{g}$ proteins were resolved by electrophoresis on a $10 \%$ or $12 \%$ NuPAGE Bis-Tris gel (Invitrogen) in MES buffer (Invitrogen) under denaturing conditions. Subsequently, the proteins were transferred onto a PVDF membrane (Millipore) using semi-dry method and blocked in $10 \%$ dry milk in TBST for $1 \mathrm{hr}$ at RT. Both primary and secondary antibodies were diluted in 5\% dry milk in TBST. Membranes were incubated with primary rabbit polyclonal anti-SOX11 antibody $(1: 1000)$ (Atlas Antibodies $\mathrm{AB}$ ) over-night at $4{ }^{\circ} \mathrm{C}$, washed and then probed with HPR conjugated donkey anti-rabbit antibody $(1: 5000)$ for 2 hours at RT (GE Healthcare). For loading control, membranes were treated with SG substrate (Vector Laboratories) before re-probing with mouse monoclonal actin antibody $(1: 5000)$ (Santa Cruz Biotechnology, Inc.) and HRP conjugated anti-mouse antibody (1:5000) (GE Healthcare Life Sciences). Blots were incubated with Western Lightning Plus-ECL enhanced chemiluminescence substrate (PerkinElmer, Inc.) for 5 min and wet developed (Kodak Developer and Fixer, Sigma Aldrich) on the Amersham Hyperfilm ECL (GE Healthcare).

Ethical permission. This study was approved by The Ethics Committee at Karolinska Institutet.

1. Bhattaram, P. et al. Organogenesis relies on SoxC transcription factors for the survival of neural and mesenchymal progenitors. Nat Commun 1, 1-12 (2010).

2. Dy, P. et al. The three SoxC proteins--Sox4, Sox11 and Sox12--exhibit overlapping expression patterns and molecular properties. Nucleic Acids Res 36, 3101-3117 (2008).

3. Schilham, M. W. et al. Defects in cardiac outflow tract formation and pro-Blymphocyte expansion in mice lacking Sox-4. Nature 380, 711-714 (1996).

4. Chen, Y.H., Gao, J., Fan, G. \& Peterson, L. C. Nuclear expression of sox11 is highly associated with mantle cell lymphoma but is independent of $\mathrm{t}(11 ; 14)(\mathrm{q} 13 ; \mathrm{q} 32)$ in non-mantle cell B-cell neoplasms. Mod Pathol 23, 105-112 (2010).

5. Dictor, M. et al. Strong lymphoid nuclear expression of SOX11 transcription factor defines lymphoblastic neoplasms, mantle cell lymphoma and Burkitt's lymphoma. Haematologica 94, 1563-1568 (2009).

6. Ek, S., Dictor, M., Jerkeman, M., Jirstrom, K. \& Borrebaeck, C. A. Nuclear expression of the non B-cell lineage Sox 11 transcription factor identifies mantle cell lymphoma. Blood 111, 800-805 (2008).

7. Wang, X. et al. The subcellular Sox 11 distribution pattern identifies subsets of mantle cell lymphoma: correlation to overall survival. Br J Haematol 143, 248-252 (2008).

8. Fernandez, V. et al. Genomic and gene expression profiling defines indolent forms of mantle cell lymphoma. Cancer Res 70, 1408-1418 (2010).

9. Nygren, L. et al. Prognostic role of SOX11 in a population-based cohort of mantle cell lymphoma. Blood 119, 4215-4223 (2012).

10. Royo, C. et al. Non-nodal type of mantle cell lymphoma is a specific biological and clinical subgroup of the disease. Leukemia [Epub ahead of print], doi: 10.1038/ leu.2012.1072. (2012)

11. Royo, C. et al. The complex landscape of genetic alterations in mantle cell lymphoma. Semin Cancer Biol 21, 322-334.

12. Gustavsson, E. et al. SOX11 expression correlates to promoter methylation and regulates tumor growth in hematopoietic malignancies. Mol Cancer 9, 187 (2010).

13. Vegliante, M. C. et al. Epigenetic activation of SOX11 in lymphoid neoplasms by histone modifications. PLoS One 6, e21382 (2011).

14. Wang, X. et al. Gene expression profiling and chromatin immunoprecipitation identify DBN1, SETMAR and HIG2 as direct targets of SOX11 in mantle cell lymphoma. PLoS One 5, e14085 (2010).

15. Ripperger, T. et al. Promoter methylation of PARG1, a novel candidate tumor suppressor gene in mantle-cell lymphomas. Haematologica 92, 460-468 (2007).

16. Schmelz, K. et al. Induction of gene expression by 5 -Aza- 2 '-deoxycytidine in acute myeloid leukemia (AML) and myelodysplastic syndrome (MDS) but not epithelial cells by DNA-methylation-dependent and -independent mechanisms. Leukemia 19, 103-111 (2005).

17. Gius, D. et al. Distinct effects on gene expression of chemical and genetic manipulation of the cancer epigenome revealed by a multimodality approach. Cancer Cell 6, 361-371 (2004).

18. Radpour, R. et al. Integrated epigenetics of human breast cancer: synoptic investigation of targeted genes, microRNAs and proteins upon demethylation treatment. PLoS One 6, e27355 (2011).

19. Huang, Y. W. et al. Epigenetic repression of microRNA-129-2 leads to overexpression of SOX4 oncogene in endometrial cancer. Cancer Res 69, 9038-9046 (2009). 
20. Shen, R., Pan, S., Qi, S., Lin, X. \& Cheng, S. Epigenetic repression of microRNA129-2 leads to overexpression of SOX4 in gastric cancer. Biochem Biophys Res Commun 394, 1047-1052 (2010)

\section{Acknowledgements}

This study was supported by grants from the Swedish Cancer Society, The Swedish Research Council, The Cancer Society in Stockholm, The Karolinska Institutet Funds and the Stockholm County Council.

\section{Author contributions}

B.S. is the principal investigator. A.M.W., M.L., X.W., F.Z. and M.K. performed experiments. P.A. and E.K. provided patient samples and clinical data. B.C. and B.S. evaluated and characterized the primary tumors. A.M.W., M.L., X.W., F.Z., B.C., M.K. and
B.S. performed data analysis. A.M.W. and B.S. wrote the manuscript. All authors critically read and approved the manuscript.

\section{Additional information}

Supplementary information accompanies this paper at http://www.nature.com/ scientificreports

Competing financial interests: The authors declare no competing financial interests. License: This work is licensed under a Creative Commons

Attribution-NonCommercial-NoDerivs 3.0 Unported License. To view a copy of this license, visit http://creativecommons.org/licenses/by-nc-nd/3.0/

How to cite this article: Wasik, A.M. et al. SOXC transcription factors in mantle cell lymphoma: the role of promoter methylation in SOX11 expression. Sci. Rep. 3, 1400; DOI:10.1038/srep01400 (2013) 